\title{
Training Practices and Injuries in Competitive Adolescent Distance Runners: A Retrospective Cross-Sectional Study.
}

\section{Robert H Mann}

University of Exeter

\section{Carly D McKay}

University of Bath

Bryan C Clift

University of Bath

\section{Craig A Williams}

University of Exeter

Alan R Barker ( $\sim$ A.R.Barker@exeter.ac.uk )

University of Exeter https://orcid.org/0000-0001-8610-5417

Research article

Keywords: Youth, Endurance, Health, Epidemiology, Track and Field

Posted Date: September 8th, 2020

DOl: https://doi.org/10.21203/rs.3.rs-60188/v1

License: (9) This work is licensed under a Creative Commons Attribution 4.0 International License. Read Full License

Version of Record: A version of this preprint was published at Frontiers in Sports and Active Living on June 24th, 2021. See the published version at https://doi.org/10.3389/fspor.2021.664632. 


\section{Abstract}

Background: Distance running is one of the most popular sports around the world. The epidemiology of running-related injuries (RRI) has been examined in adults, but only a few studies have focused on adolescent distance runners. Therefore, the purpose of this study was 1) to examine the training practices (frequency, volume, and intensity) and descriptive epidemiology of RRI (risks, rates, sites, and severity) in competitive adolescent distance runners (13-18 years) in England, and 2) to explore potential correlates (risk factors) of RRI.

Methods: A cross-sectional study design was used. Adolescent distance runners $(n=113)$ were recruited from England Athletics affiliated athletics clubs. Participants voluntarily completed an online questionnaire between April and December 2018. At the point of completion, responses were based on the participant's previous 12-months of distance running participation. Injury incidence proportions (IP) and incidence rates (IR) were calculated. Potential correlates of RRI were estimated using an odds ratio (OR) and 95\% confidence intervals (Cl).

Results: The injury IP was 122/100 participants/year (95\% Cl: 113 to 138). The injury IR was 6.3/1000 participation hours ( $95 \% \mathrm{Cl}: 5.3$ to 7.4 ). The most common injury sites were the knee, foot/toes, and lower leg; primarily caused by overuse. Exploratory univariate analyses showed a larger number of training sessions per week (volume) is associated with a lower risk of RRI (OR $=0.71,95 \% \mathrm{Cl}: 0.53$ to 0.94$)$, and that a higher level of specialisation is associated with a lower risk of time loss injury $(\mathrm{OR}=0.26,95 \% \mathrm{Cl}$ : 0.11 to 0.63$)$.

Conclusions: Injury is common in adolescent distance runners, aligned with adult-based research. These data provide guidance for the development of appropriate injury prevention interventions.

\subsection{Background}

Distance running is one of the most popular sports around the world [1]. While distance running is associated with numerous health benefits [2], adult-based research shows that participation is also associated with adverse health outcomes such as injury [3, 4]. Injuries sustained from distance running are usually situated in the lower extremity, with the lower leg, knee, and foot/toes being the most common sites [3,5]. Systematic reviews highlight different injury rates ranging from 2.5 to 33.0 injuries per 1000 hours of participation [4]. and incidence proportions between $3.2 \%$ and $79.3 \%$ in adult distance runners [3, 6]. The variation in study results could be explained by differences in research methodology [7]. Regardless, there is less research that has investigated injuries in adolescent distance runners (1318 years) [8-14]. This is an issue given that distance running is the second most prevalent sport amongst adolescents in England [15], whereby an understanding of common running-related injuries (RRI) during maturation is important for supporting long-term athletic development. This is supported by a recent International Olympic Committee (IOC) consensus paper which recommends that youth athletic 
development should be evidence-informed [16]. Also, the few available studies usually focus on adolescent track and field (athletics), only including distance running as a sub-sample $[13,14,17,18]$.

Although excelling as an adolescent track and field athlete is unessential for success as a senior athlete $[19,20]$, research has shown that $90 \%$ of youth athletes in the United Kingdom decide to specialise in selected events from an early age (13-14 years) [21]. Research has highlighted that youth sport specialisation is positively associated with injury history $[22,23]$. Therefore, the trend towards early sport specialisation is a concern in relation to adolescent distance runners [24], whereby 'success' for endurance athletes is usually attributed to consistent (and monotonous) training intensities, durations, and frequencies [25]. These demanding training practices may contribute to RRI [26], yet, little is known about the training practices of adolescent distance runners, or whether age, growth, and maturation related issues, contribute to RRI in this given population. A better understanding of the training practices and RRI of adolescent distance runners can inform the development of injury prevention interventions. Therefore, the primary purpose of this study was to examine the training practices (frequency, volume, and intensity) and provide descriptive epidemiology of RRI (risks, rates, sites, and severity) in competitive adolescent distance runners in England. The secondary purpose was to investigate potential correlates (risk factors) of RRI in this population.

\subsection{Methods}

\subsection{Study Design:}

This was a cross-sectional study based on the completion of an online questionnaire. Data collection took place between April and December 2018.

\subsection{Participants:}

Participants were included if they were a member of an England Athletics affiliated athletics club, aged between 13 and 18 years, and training for and/or competing in a distance running event (800 $\mathrm{m}$ up to $10,000 \mathrm{~m}$, including steeplechase). Participants were excluded if they were unable to fully understand the study procedures and/or failed to complete the full questionnaire. Using convenience sampling, participants were recruited directly from England Athletics affiliated athletics clubs, with study information being distributed via face-to-face meetings, email, and/or social media. Due to the nature of participant recruitment, it was not possible to determine the total number of athletes approached to take part in this study. Written parental consent and participant assent were obtained before completion of the questionnaire. Ethics approval was granted by the institutional ethics committee (171206/B/02).

\subsection{Data Collection Procedure}

\subsubsection{Study Questionnaire:}

Participants completed the questionnaire via the online platform (Qualtrics XM., Provo, Utah, USA), which is compatible with computers and mobile devices. The questionnaire included sections related to 
background demographics (e.g. date of birth), performance history (e.g. event preferences), training practices (e.g. weekly training volume), medical information (e.g. history of injury and/or 'pains or discomforts'), and the Athlete Identity Measurement Scale [27]. A copy of the questionnaire has been uploaded as a supplementary file. Development of the questionnaire was based on methods used in previous studies $[12,13,28]$. Key stakeholders were also involved in the development of the study questionnaire to ensure it was suitable for the target audience. This included adolescent distance runners, parents, coaches, and physiotherapists $(n=12)$.

\subsubsection{Injury Definition and Classification:}

The primary outcome measure was RRI, defined as any physical complaint that resulted from distance running participation (i.e. training or competition), irrespective of the need for medical attention or time loss from distance running activities. This definition of RRI was named "all RRI" and included selfreported 'pains or discomforts.' RRI needed to have occurred during the 12-months immediately preceding questionnaire completion. This definition was adapted from an IOC consensus statement on load in sport and injury risk [29], and from a consensus statement on injury and illness research design in studies on track and field [30]. RRI that required medical attention and/or resulted in time loss from full distance running participation were also recorded. Time loss injuries were classified according to their level of severity: none ( 0 days), slight ( 1 day), minimal (2-3 days), mild (4-7 days), moderately serious (8-28 days), serious ( $>28$ days -6 months) or long-term (> 6 months) [30]. Medical attention injuries were classified as any RRI that involved the assessment of a participant's condition by a medical or healthcare practitioner. Both broad and narrow definitions of RRI were used in order to capture the full range of RRI [31]. Although mode (gradual onset/sudden onset) and cause (traumatic/overuse) of RRI were selfreported by participants, this excluded any self-reported 'pains or discomforts,' as they were not classified according to these outcomes.

\subsubsection{Intrinsic and Extrinsic Injury Risk Factors:}

Data collection included the assessment of potential intrinsic and extrinsic injury risk factors. These risk factors were selected based on previous youth sport injury studies [23, 32, 33], and adult-based distance running injury studies [3]. Potential intrinsic risk factors included sex, age-group, stature, body mass, body mass index (BMI), maturity timing, maturity tempo, training age, performance level, level of specialisation, and history of previous injury. Potential extrinsic risk factors included event preferences, training practices (volume and intensity), use of a coach, inclusion of a warm-up or cool-down, and inclusion of strength and conditioning as part of their weekly training.

Age-group was categorised according to age ranges: 13-14 years (U15), 15-16 years (U17) and 1718 years (U19). BMI was divided into three subgroups by applying age and sex specific cut points (i.e. underweight, normal, and overweight/obese) [34, 35]. To estimate maturity timing and tempo, each participant's age at peak height velocity (PHV) was determined by applying sex-specific maturity offset equations [36]. Subsequently, maturity timing and tempo were categorised according to previous research [37]. Training age (i.e. number of years participating in distance running) and current performance level 
(i.e. club, country, regional, national, international) were self-reported. Level of specialisation was categorised as low, moderate, or high according to how participants responded to the following questions: "Is distance running your main sport?", "Have you quit other sports in order to focus on distance running?", and "How many months of the past year did you participate in distance running?" [38, 39]. History of previous injury was self-reported by participants, defined as an RRI that preceded the 12month retrospective period of data collection (calculated from day of questionnaire completion).

To establish event preference, participants self-reported the number of distance running events that they trained for and/or competed in. Selecting one event was categorised as a "single event preference", while two or more events was categorised as a "multi event preference". Training practices were self-reported as hours per week and days per week. These variables were also used to calculate injury incidence rates (IR). To calculate internal training load (ITL), each participant self-reported their most recent training week, including the duration ( $\mathrm{min}$ ), distance $(\mathrm{km})$, session type (i.e. interval session), and rating of perceived exertion (RPE) for each of their training sessions. Weekly session RPE (SRPE) was subsequently calculated, having been validated as a measure of ITL in adolescent distance runners [40]. Use of a coach, inclusion of a warm-up/cool-down, and inclusion of a strength and conditioning programme were categorised via binary (yes/no) self-report responses.

\subsection{Statistical Methods}

The statistical software SPSS (version 26.0; IBM., Chicago, USA) and an Excel spreadsheet (Microsoft., Redmond, USA) were used to conduct the analysis. An a priori target sample size of 151 participants was calculated based on a logistic regression analysis identifying an effect of 1.75 for the odds ratio (OR) between the predictor variables and RRI with an $80 \%$ power and alpha of 0.05 [3]. Incidence proportion $(I P)$, reported as a percentage (\%), was calculated to determine the average probability that a participant would sustain at least one injury during the 12-month study period. Clinical incidence, reported per 100 participants/year, was calculated to determine the proportion of participants who experienced RRI during the 12-month study period. IR was calculated to establish the number of RRI per participant/1000 hours of distance running participation. To compare the relative risk between male and female participants, risk ratios (related to IP) and rate ratios (related to IR) were calculated. When reported, male participants were used as the referent group, meaning that the higher or lower risk/rate is related to the male participants [41]. 95\% confidence intervals (CI) were calculated for IP and IR [42], in addition to the risk and rate ratios [43]. Median and interquartile range (IQR) statistics were calculated for multiple RRI and time loss RRI. Means and standard deviations (SD) were calculated for continuous variables. Percentages (\%) were calculated for categorical variables. Sex differences were analysed using independent samples t-tests for continuous variables and Chi-squared tests $\left(\mathrm{X}^{2}\right)$ for categorical variables. Differences between age-group training practices were analysed using one-way ANOVA. Pairwise comparisons were subsequently used to explore differences between age-groups. Statistical significance was set at an alpha level of 0.05 , and effect sizes for mean comparisons were described using Cohen's thresholds (small $=0.2$, medium $=0.5$, large $=0.8)[44]$. 
Injury sites were described using frequencies and proportions (\%). Using RRI as the dependent variable (including time loss and medical attention definitions), exploratory univariate binary logistic regression analysis was conducted using the previously defined intrinsic and extrinsic injury risk factors, chosen according to previous research $[3,23,32,33]$. Linearity and overdispersion assumptions were assessed, with risk factors being excluded from analysis if assumptions were violated. Based on the limited sample size, no exploratory multivariate analysis was conducted.

\subsection{Results}

\subsection{Descriptive Characteristics:}

A total of 115 adolescent distance runners ( 65 female) volunteered to participate in this study and completed the questionnaire. Two participants were excluded from the results as they were not members of an England Athletics affiliated Club, resulting in a sample of 113 adolescent distance runners (64 female). Participant descriptive characteristics are shown in Table 1 with sex differences reported. Agegroup (years) based training practices are presented in Table 2. ANOVA revealed a significant age-group effect for sessions per week $(p=0.012)$ with the 13-14 age-group reporting fewer sessions per week than the $17-18$ age-group $(p=0.013)$. 
Table 1

Descriptive characteristics of the participants (data presented as mean and SD, unless otherwise stated)

\begin{tabular}{|c|c|c|c|c|c|}
\hline Characteristic & $\begin{array}{l}\text { Overall (n } \\
=113 \text { ) }\end{array}$ & $\begin{array}{l}\text { Male (n } \\
=49)\end{array}$ & $\begin{array}{l}\text { Female ( } \mathrm{n} \\
=64)\end{array}$ & Value & $\begin{array}{l}\text { Effect } \\
\text { Size }\end{array}$ \\
\hline Age, years & $15.9(1.5)$ & $\begin{array}{l}15.9 \\
(1.5)\end{array}$ & $16.0(1.6)$ & 0.65 & 0.06 \\
\hline Training age, years & $5.6(2.4)$ & $5.6(2.1)$ & $5.7(2.5)$ & 0.77 & 0.04 \\
\hline Age-group (N, \%): & & & & $\begin{array}{l}x^{2}= \\
0.94\end{array}$ & \\
\hline $13-14$ years & $36(32 \%)$ & $\begin{array}{l}16 \\
(33 \%)\end{array}$ & $20(31 \%)$ & & \\
\hline $15-16$ years & $43(38 \%)$ & $\begin{array}{l}19 \\
(39 \%)\end{array}$ & $24(38 \%)$ & & \\
\hline $17-18$ years & $34(30 \%)$ & $\begin{array}{l}14 \\
(29 \%)\end{array}$ & $20(31 \%)$ & & \\
\hline Stature, $\mathrm{cm}$ & $\begin{array}{l}168.8 \\
(8.6)\end{array}$ & $\begin{array}{l}173.7 \\
(8.3)\end{array}$ & $\begin{array}{l}165.0 \\
(6.9)\end{array}$ & $\begin{array}{l}< \\
0.01\end{array}$ & 1.15 \\
\hline Body mass, kg & $52.8(8.7)$ & $\begin{array}{l}57.4 \\
(9.4)\end{array}$ & $49.3(6.0)$ & $\dot{0} .01$ & 1.06 \\
\hline BMI cut points $(\mathrm{N}, \%)$ : & & & & $\begin{array}{l}x^{2}= \\
0.02\end{array}$ & \\
\hline Underweight & $32(28 \%)$ & $8(16 \%)$ & $24(38 \%)$ & & \\
\hline Normal & $80(71 \%)$ & $\begin{array}{l}40 \\
(82 \%)\end{array}$ & $40(62 \%)$ & & \\
\hline Overweight & $1(1 \%)$ & $1(2 \%)$ & $0(0 \%)$ & & \\
\hline Maturity timing $(\mathrm{N}, \%)$ : & & & & $\begin{array}{l}x^{2}< \\
0.01\end{array}$ & \\
\hline Pre-PHV & $0(0 \%)$ & $0(0 \%)$ & $0(0 \%)$ & & \\
\hline At-PHV & $15(13 \%)$ & $\begin{array}{l}12 \\
(24 \%)\end{array}$ & $3(5 \%)$ & & \\
\hline Post-PHV & $98(87 \%)$ & $\begin{array}{l}37 \\
(76 \%)\end{array}$ & $61(95 \%)$ & & \\
\hline Maturity tempo (N, \%): & & & & $\begin{array}{l}x^{2}= \\
0.11\end{array}$ & \\
\hline Early & $0(0 \%)$ & $0(0 \%)$ & $0(0 \%)$ & & \\
\hline Average & $102(90 \%)$ & $\begin{array}{l}47 \\
(96 \%)\end{array}$ & $55(86 \%)$ & & \\
\hline
\end{tabular}




\begin{tabular}{|c|c|c|c|c|c|}
\hline Characteristic & $\begin{array}{l}\text { Overall ( } \mathrm{n} \\
=113 \text { ) }\end{array}$ & $\begin{array}{l}\text { Male }(n \\
=49)\end{array}$ & $\begin{array}{l}\text { Female }(n \\
=64)\end{array}$ & Value & $\begin{array}{l}\text { Effect } \\
\text { Size }\end{array}$ \\
\hline Late & $11(10 \%)$ & $2(4 \%)$ & $9(14 \%)$ & & \\
\hline Current performance level $(\mathrm{N}, \%)$ : & & & & $\begin{array}{l}x^{2}= \\
0.62\end{array}$ & \\
\hline Club & $23(20 \%)$ & $\begin{array}{l}11 \\
(22 \%)\end{array}$ & $12(19 \%)$ & & \\
\hline County & $25(22 \%)$ & $8(16 \%)$ & $17(27 \%)$ & & \\
\hline Regional & $19(17 \%)$ & $\begin{array}{l}10 \\
(20 \%)\end{array}$ & $9(14 \%)$ & & \\
\hline National & $41(36 \%)$ & $\begin{array}{l}17 \\
(35 \%)\end{array}$ & $24(37 \%)$ & & \\
\hline International & $5(4 \%)$ & $3(6 \%)$ & $2(3 \%)$ & & \\
\hline Level of specialisation $(\mathrm{N}, \%)$ : & & & & $\begin{array}{l}x^{2}= \\
0.61\end{array}$ & \\
\hline Low & $9(8 \%)$ & $5(10 \%)$ & $4(6 \%)$ & & \\
\hline Moderate & $39(35 \%)$ & $\begin{array}{l}15 \\
(31 \%)\end{array}$ & $24(38 \%)$ & & \\
\hline High & $65(58 \%)$ & $\begin{array}{l}29 \\
(59 \%)\end{array}$ & $36(56 \%)$ & & \\
\hline Event preferences $(\mathrm{N}, \%)$ : & & & & $\begin{array}{l}x^{2}= \\
0.46\end{array}$ & \\
\hline One (single) & $8(7 \%)$ & $2(4 \%)$ & $6(9 \%)$ & & \\
\hline Two or more (multiple) & $105(93 \%)$ & $\begin{array}{l}47 \\
(96 \%)\end{array}$ & $58(91 \%)$ & & \\
\hline \multicolumn{6}{|l|}{ Training practices: } \\
\hline Sessions per week & $4.7(1.5)$ & $4.9(1.6)$ & $4.6(1.5)$ & 0.28 & 0.20 \\
\hline Weeks per month & $4.0(0.2)$ & $4.0(0.1)$ & $3.9(0.3)$ & 0.15 & 0.27 \\
\hline Months per year & $11.0(2.1)$ & $\begin{array}{l}11.2 \\
(1.7)\end{array}$ & $10.8(2.3)$ & 0.31 & 0.19 \\
\hline Average session duration (min) & $\begin{array}{l}57.6 \\
(19.6)\end{array}$ & $\begin{array}{l}56.1 \\
(17.2)\end{array}$ & $\begin{array}{l}58.8 \\
(21.3)\end{array}$ & 0.46 & 0.14 \\
\hline Average RPE per session (AU) & $6.4(1.4)$ & $6.2(1.3)$ & $6.5(1.5)$ & 0.25 & 0.21 \\
\hline Average sRPE per session (AU) & $\begin{array}{l}391.9 \\
(177.7)\end{array}$ & $\begin{array}{l}373.7 \\
(158.7)\end{array}$ & $\begin{array}{l}405.9 \\
(191.1)\end{array}$ & 0.34 & 0.18 \\
\hline
\end{tabular}




\begin{tabular}{|c|c|c|c|c|c|}
\hline Characteristic & $\begin{array}{l}\text { Overall (n } \\
=113)\end{array}$ & $\begin{array}{l}\text { Male (n } \\
=49)\end{array}$ & $\begin{array}{l}\text { Female (n } \\
=64)\end{array}$ & $p_{\text {Value }}$ & $\begin{array}{l}\text { Effect } \\
\text { Size }\end{array}$ \\
\hline Have a coach $(\mathrm{N}, \%)$ : & & & & $\begin{array}{l}x^{2}= \\
0.63\end{array}$ & \\
\hline Yes & $109(96 \%)$ & $\begin{array}{l}48 \\
(98 \%)\end{array}$ & $61(95 \%)$ & & \\
\hline No & $4(4 \%)$ & $1(2 \%)$ & $3(5 \%)$ & & \\
\hline Inclusion of a warm-up ( $\mathrm{N}, \%)$ : & & & & $\begin{array}{l}x^{2}= \\
0.43\end{array}$ & \\
\hline Yes & $112(99 \%)$ & $\begin{array}{l}48 \\
(98 \%)\end{array}$ & $64(100 \%)$ & & \\
\hline No & $1(1 \%)$ & $1(2 \%)$ & $0(0 \%)$ & & \\
\hline Inclusion of a cool-down ( $\mathrm{N}, \%)$ : & & & & $\begin{array}{l}\mathrm{X}^{2}= \\
0.19\end{array}$ & \\
\hline Yes & $111(98 \%)$ & $\begin{array}{l}47 \\
(96 \%)\end{array}$ & $64(100 \%)$ & & \\
\hline No & $2(2 \%)$ & $2(4 \%)$ & $0(0 \%)$ & & \\
\hline $\begin{array}{l}\text { Inclusion of a strength and conditioning } \\
\text { programme }(\mathrm{N}, \%) \text { : }\end{array}$ & & & & $\begin{array}{l}x^{2}= \\
0.11\end{array}$ & \\
\hline Yes & $89(79 \%)$ & $\begin{array}{l}35 \\
(71 \%)\end{array}$ & $54(84 \%)$ & & \\
\hline No & $24(21 \%)$ & $\begin{array}{l}14 \\
(29 \%)\end{array}$ & $10(16 \%)$ & & \\
\hline \multicolumn{6}{|c|}{$\begin{array}{l}\text { Abbreviations: N, number; cm, centimetres; kg, kilograms; BMI, Body Mass Index; PHV, Peak Height } \\
\text { Velocity; min, minutes; RPE, Rating of Perceived Exertion; SRPE, Session Rating of Perceived Exertion; } \\
\text { AU, Arbitrary Units. }\end{array}$} \\
\hline \multicolumn{6}{|c|}{ NB: Due to rounding, not all numbers add up correctly. } \\
\hline
\end{tabular}


Table 2

Training practices according to age-group (data presented as mean and SD)

\begin{tabular}{|c|c|c|c|}
\hline Training practices & $\begin{array}{l}13-14 \text { years }(n= \\
36)\end{array}$ & $\begin{array}{l}15-16 \text { years }(n= \\
43)\end{array}$ & $\begin{array}{l}\text { 17-18 years }(n= \\
34)\end{array}$ \\
\hline Sessions per week * & $4.1(1.2)$ & $4.9(1.5)$ & $5.1(1.8)$ \\
\hline Weeks per month & $3.9(0.2)$ & $3.9(0.3)$ & $3.9(0.2)$ \\
\hline Months per year & $11.3(1.6)$ & $10.9(1.9)$ & $10.6(2.7)$ \\
\hline $\begin{array}{l}\text { Average session duration } \\
\text { (min) }\end{array}$ & $58.5(20.5)$ & $53.7(21.0)$ & $61.7(16.1)$ \\
\hline Average RPE per session (AU) & $6.3(1.3)$ & $6.6(1.5)$ & $6.3(1.3)$ \\
\hline $\begin{array}{l}\text { Average sRPE per session } \\
\text { (AU) }\end{array}$ & $379.6(150.6)$ & $400.8(198.0)$ & $393.8(181.6)$ \\
\hline \multicolumn{4}{|c|}{$\begin{array}{l}\text { Abbreviations: RPE, Rating of Perceived Exertion; SRPE, Session Rating of Perceived Exertion; AU, } \\
\text { Arbitrary Units. NB: Due to rounding, not all numbers add up correctly. }{ }^{*} p<0.05 \text { between groups. }\end{array}$} \\
\hline
\end{tabular}

Seventy-seven (68\%) participants sustained at least one new RRI during the 12-month study period, including self-reported 'pains or discomforts.' Thirty-six (47\%) reported multiple RRI (median: 2; IQR: 1). The total number of RRI sustained was 142, resulting in an IP of $68 \%$ (95\% Cl: $60-77 \%)$, and a clinical incidence of $126 / 100$ participants/year ( $95 \%$ Cl: 113 to 138). The overall IR was 6.3/1000 participation hours ( $95 \% \mathrm{Cl}: 5.3$ to 7.4$)$. Eleven (10\%) participants had a history of previous RRI.

Thirty-one (63\%) male participants sustained $60 \mathrm{RRI}(\mathrm{IP}=63 \%$ (95\% Cl: 60-77\%); clinical incidence= 122/100 participants/year ( $95 \% \mathrm{Cl}$ : 104 to 141$)$ ) compared with forty-six $(72 \%)$ female participants who sustained $82 \mathrm{RRI}(\mathrm{IP}=72 \%$ (95\% Cl: 61-83\%); clinical incidence $=128 / 100$ participants/year (95\% Cl: 111 to 145$)$ ), resulting in a risk ratio of 0.88 (95\% Cl: 0.68 to 1.14$)$. The IR for male participants was $5.9 / 1000$ participation hours ( $95 \% \mathrm{Cl}: 4.6$ to 7.6$)$, and for female participants it was 6.6/1000 participation hours (95\% Cl: 5.3 to 8.2$)$, resulting in a rate ratio of 0.89 (95\% Cl: 0.68 to 1.33$)$.

\subsubsection{Time Loss RRI:}

Sixty-five (58\%) participants sustained at least one new time loss RRI during the 12-month study period, including self-reported 'pains or discomforts.' A total of $112(79 \%)$ RRI resulted in at least one day of time loss from distance running, resulting in an IP of $58 \%$ (95\% Cl: $48-67 \%)$, and a clinical incidence of 99/100 participants/year (95\% Cl: 97 to 101). The IR was 5.0/1000 participation hours (95\% Cl: 4.1 to 6.0). The median amount of time loss was seven days (IQR: 34). 
Twenty-nine (59\%) male participants sustained $45 \mathrm{RRI}(\mathrm{IP}=59 \%$ (95\% Cl: $45-73 \%)$; clinical incidence = 92/100 participants/year ( $95 \% \mathrm{Cl}: 82$ to 100)) compared with thirty-six (56\%) female participants who sustained 67 RRI (IP = 56\% (95\% Cl: 44-68\%); clinical incidence = 105/100 participants/year (95\% Cl: 98 to 111$)$, resulting in a risk ratio of 1.05 (95\% Cl: 0.77 to 1.45 ). The IR for male participants was $4.4 / 1000$ participation hours (95\% Cl: 3.3 to 5.9), and for female participants it was 5.4/1000 participation hours ( $95 \% \mathrm{Cl}: 4.3$ to 6.9$)$, resulting in a rate ratio of $0.82(95 \% \mathrm{Cl}: 0.63$ to 1.34$)$. Figure 1 represents the proportion of "all RRI" that resulted in time loss according to severity and sex.

\subsubsection{Medical Attention RRI:}

Forty-four (39\%) participants sustained at least one new RRI during the 12-month retrospective study period that required medical attention. These participants received medical attention for a total of $59 \mathrm{RRI}$, resulting in an IP of $39 \%$ (95\% Cl: $30-48 \%$ ), and a clinical incidence of 52/100 participants/year ( $95 \% \mathrm{Cl}$ : 41 to 63 ). The IR was $2.61 / 1000$ participation hours (95\% Cl: 2.59 to 2.63 ).

Fifteen (31\%) male participants sustained 19 RRI (IP = 31\% (95\% Cl: 18-44\%); clinical incidence = 39/100 participants/year (95\% Cl: 22 to 56$)$ ) compared with twenty-nine (45\%) female participants who sustained $40 \mathrm{RRI}(\mathrm{IP}=45 \%$ (95\% Cl: 33-58\%); clinical incidence $=63 / 100$ participants/year (95\% Cl: 49 to 76)), resulting in a risk ratio of $0.68(95 \% \mathrm{Cl}: 0.41$ to 1.11$)$. The IR for male participants was $1.9 / 1000$ participation hours ( $95 \% \mathrm{Cl}: 1.84$ to 1.90), and for female participants it was 3.2/1000 participation hours ( $95 \% \mathrm{Cl}: 3.19$ to 3.26$)$, resulting in a rate ratio of 0.58 (95\% Cl: 0.46 to 1.36$)$. Medical attention by a physiotherapist was the most common type of treatment self-reported (92\%).

\subsection{Injury Characteristics:}

Injury sites are summarised in Table 3. Excluding self-reported 'pains or discomfort', there were a total of 76 self-reported RRI, representing $54 \%$ of all RRI. Of these 76 RRI, the most commonly reported mode of injury was gradual onset (51\%) and the most commonly reported cause of injury was overuse (84\%). 
Table 3

Self-reported RRI by body region and area.

\begin{tabular}{|c|c|c|}
\hline Injury Site & Frequency (\%) & IR/1000 h (95\% Cl) \\
\hline Lower Extremity & $131(92)$ & 5.8 (4.9 to 6.9$)$ \\
\hline Knee & $31(22)$ & 1.4 (1.0 to 2.0$)$ \\
\hline Foot/toe & $23(16)$ & 1.0 (0.7 to 1.5$)$ \\
\hline Lower leg & $22(16)$ & 1.0 (0.6 to 1.5$)$ \\
\hline Thigh & $17(12)$ & 0.8 (0.5 to 1.2$)$ \\
\hline Ankle & $14(10)$ & 0.6 (0.4 to 1.1$)$ \\
\hline Hip & $11(8)$ & 0.5 (0.3 to 0.9$)$ \\
\hline Achilles tendon & $7(5)$ & 0.3 (0.2 to 0.7$)$ \\
\hline Groin & $6(4)$ & 0.3 (0.2 to 0.6$)$ \\
\hline Upper Extremity & $1(1)$ & $0.1(0.0$ to 0.3$)$ \\
\hline Elbow & $1(1)$ & 0.1 (0.0 to 0.3$)$ \\
\hline Head and Trunk & $10(7)$ & $0.4(0.2$ to 0.8$)$ \\
\hline Lumbar spine/lower back & $8(6)$ & $0.4(0.2$ to 0.7$)$ \\
\hline Pelvis/sacrum/buttock & $2(1)$ & 0.1 (0.0 to 0.4$)$ \\
\hline Total & $142(100)$ & 6.3 (5.3 to 7.4$)$ \\
\hline
\end{tabular}


Table 4

Exploratory univariate logistic regression analyses

\begin{tabular}{|c|c|c|c|c|}
\hline & $\begin{array}{l}\text { IR/1000 h } \\
(95 \% \mathrm{Cl})\end{array}$ & $\begin{array}{l}\text { All RRI } \\
\text { OR (95\% } \\
\text { Cl) }\end{array}$ & $\begin{array}{l}\text { Time loss } \\
\text { RRI } \\
\text { OR ( } 95 \% \\
\text { Cl) }\end{array}$ & $\begin{array}{l}\text { Medical attention } \\
\text { RRI OR ( } 95 \% \mathrm{Cl})\end{array}$ \\
\hline Total number of RRI & & 142 & 112 & 59 \\
\hline \multicolumn{5}{|l|}{ Intrinsic Risk Factors } \\
\hline \multicolumn{5}{|l|}{ Sex: } \\
\hline Male & $\begin{array}{l}5.9 \text { ( } 4.6 \text { to } \\
7.6)\end{array}$ & 1 & 1 & 1 \\
\hline Female & $\begin{array}{l}6.6(5.3 \text { to } \\
8.2)\end{array}$ & $\begin{array}{l}0.76(0.34 \\
\text { to } 1.71)\end{array}$ & $\begin{array}{l}1.86(0.87 \\
\text { to } 3.96)\end{array}$ & $0.64(0.30$ to 1.38$)$ \\
\hline \multicolumn{5}{|l|}{ Age-group: } \\
\hline 13-14 years & $\begin{array}{l}5.7(4.1 \text { to } \\
7.9)\end{array}$ & 1 & 1 & 1 \\
\hline $15-16$ years & $\begin{array}{l}6.2(4.7 \text { to } \\
8.2)\end{array}$ & $\begin{array}{l}1.57(0.57 \\
\text { to } 4.36)\end{array}$ & $\begin{array}{l}0.64(0.25 \\
\text { to } 1.63)\end{array}$ & 1.59 (0.59 to 4.26$)$ \\
\hline $17-18$ years & $\begin{array}{l}6.8 \text { (5.3 to } \\
8.9)\end{array}$ & $\begin{array}{l}1.34(0.50 \\
\text { to } 3.62)\end{array}$ & $\begin{array}{l}0.48(0.19 \\
\text { to } 1.20)\end{array}$ & 0.88 (0.36 to 2.20$)$ \\
\hline Stature (cm) & N/A & $\begin{array}{l}1.00(0.96 \\
\text { to } 1.05)\end{array}$ & $\begin{array}{l}1.03(0.98 \\
\text { to } 1.07)\end{array}$ & 0.97 (0.93 to 1.01$)$ \\
\hline Weight (kg) & $\mathrm{N} / \mathrm{A}$ & $\begin{array}{l}0.97(0.93 \\
\text { to } 1.02)\end{array}$ & $\begin{array}{l}1.01(0.97 \\
\text { to } 1.05)\end{array}$ & 0.99 (0.95 to 1.03$)$ \\
\hline \multicolumn{5}{|l|}{ Maturity timing: } \\
\hline At-PHV & $\begin{array}{l}5.3(3.1 \text { to } \\
8.9)\end{array}$ & 1 & 1 & 1 \\
\hline Post-PHV & $\begin{array}{l}6.4(5.4 \text { to } \\
7.6)\end{array}$ & $\begin{array}{l}0.75(0.22 \\
\text { to } 2.54)\end{array}$ & $\begin{array}{l}0.89(0.29 \\
\text { to } 2.69)\end{array}$ & 1.32 (0.42 to 4.16$)$ \\
\hline \multicolumn{5}{|l|}{ Maturity tempo: } \\
\hline Average & $\begin{array}{l}6.3 \text { (5.3 to } \\
7.5)\end{array}$ & 1 & 1 & 1 \\
\hline Late & $\begin{array}{l}6.2(3.8 \text { to } \\
9.9)\end{array}$ & $\begin{array}{l}2.25(0.46 \\
\text { to } 11.00)\end{array}$ & $\begin{array}{l}0.58(0.17 \\
\text { to } 2.04)\end{array}$ & 0.56 (0.14 to 2.23$)$ \\
\hline Training age, years & $\mathrm{N} / \mathrm{A}$ & $\begin{array}{l}0.93(0.79 \\
\text { to } 1.11)\end{array}$ & $\begin{array}{l}1.05(0.90 \\
\text { to } 1.23)\end{array}$ & $1.08(0.92$ to 1.27$)$ \\
\hline Level of specialisation & & & & \\
\hline
\end{tabular}


Low

Moderate

High
$19.8(11.8 \quad 1$ to 33.3$)$
6.6 (5.0 to $\quad 0.26(0.03 \quad 0.69(0.17 \quad 0.53(0.13$ to 2.17$)$

8.7) to 2.23) to 2.79)
1

$\begin{array}{lll}1.17(0.51 & \mathbf{0 . 2 6}(0.11 & 1.33(0.58 \text { to } 3.06) \\ \text { to } 2.71) & \text { to } 0.63) *\end{array}$

\section{Extrinsic Risk Factors}

Event preferences:

One (single)

Two or more (multiple)
5.5 (4.4 to

6.8)

Event preferences:

.

Training practices:

\begin{tabular}{|c|c|c|c|c|}
\hline Sessions per week & N/A & $\begin{array}{l}0.71(0.53 \\
\text { to } 0.94) *\end{array}$ & $\begin{array}{l}0.98(0.77 \\
\text { to } 1.25)\end{array}$ & 0.85 (0.66 to 1.09$)$ \\
\hline Average session duration (min) & N/A & $\begin{array}{l}0.99(0.97 \\
\text { to } 1.01)\end{array}$ & $\begin{array}{l}1.01(0.99 \\
\text { to } 1.03)\end{array}$ & 1.01 (0.99 to 1.03$)$ \\
\hline Weeks per month & N/A & $\begin{array}{l}2.43(0.27 \\
\text { to } 21.60)\end{array}$ & $\begin{array}{l}1.51(0.27 \\
\text { to } 8.59)\end{array}$ & $0.77(0.14$ to 4.14$)$ \\
\hline Months per year & N/A & $\begin{array}{l}1.15(0.91 \\
\text { to } 1.46)\end{array}$ & $\begin{array}{l}1.05(0.88 \\
\text { to } 1.27)\end{array}$ & 1.08 (0.90 to 1.29$)$ \\
\hline
\end{tabular}

Have a coach:

\begin{tabular}{|c|c|c|c|c|}
\hline Yes & $\begin{array}{l}6.2(5.2 \text { to } \\
7.3)\end{array}$ & 1 & 1 & 1 \\
\hline No & $\begin{array}{l}10.9(4.9 \\
\text { to } 24.1)\end{array}$ & $\begin{array}{l}1.42(0.14 \\
\text { to } 14.13)\end{array}$ & $\begin{array}{l}0.23(0.02 \\
\text { to } 2.33)\end{array}$ & 1.59 (0.22 to 11.76$)$ \\
\hline
\end{tabular}

Inclusion of a strength and

conditioning programme:

\begin{tabular}{|c|c|c|c|c|}
\hline Yes & $\begin{array}{l}6.0(5.0 \text { to } \\
7.2)\end{array}$ & 1 & 1 & 1 \\
\hline No & $\begin{array}{l}7.6(5.3 \text { to } \\
11.0)\end{array}$ & $\begin{array}{l}1.53(0.55 \\
\text { to } 4.24)\end{array}$ & $\begin{array}{l}1.30(0.52 \\
\text { to } 3.28)\end{array}$ & $0.74(0.29$ to 1.90$)$ \\
\hline
\end{tabular}

Abbreviations: IR, Incidence Rate; RRI, running-related injuries; h, hours; $\mathrm{Cl}$, Confidence Intervals; OR, Odds Ratio; cm, centimetres; kg, kilograms; PHV, Peak Height Velocity; min, minutes. NB: Self-reported 'pains or discomforts' are included in each of the RRI outcomes. The following risk factors were excluded from analyses due to violation of statistical assumptions: body mass index cut points; current performance level; average weekly rating of perceived exertion; average weekly session rating of perceived exertion; use of warm-up; use of cool-down. * $p<0.05$. 


\subsection{Potential Correlates:}

Exploratory univariate analyses are presented in Table 4. Results suggested that the number of training sessions per week was inversely associated with risk of injury, when calculated for all RRI. In relation to time loss injuries, a higher level of specialisation was associated with a lower risk of injury, when compared to a lower level of specialisation.

\subsection{Discussion}

This study provides novel insights into the training practices and RRI in a population of competitive adolescent distance runners. The key findings were that: 1) the number of training sessions per week (frequency) increased with chronological age; 2 ) for "all RRI," the IP was 126/100 participants/year (95\% Cl: 113 to 138 ), and the IR was $6.3 / 1000$ participation hours (95\% Cl: 5.3 to 7.4$) ; 3$ ) the most common injury sites were the knee, foot/toe, and lower leg, with overuse being the most common cause of injury; and 4) "sessions per week" and a "higher level of specialisation" were associated with a lower risk of all RRI and time loss RRI, respectively.

\subsection{Training Practices:}

The results highlight that the number of training sessions per week (frequency) are significantly different between age-groups, with a higher number of weekly sessions being recorded in the older 17-18 agegroup, compared to the 13-14 age-group. This finding supports that reported in a cohort of elite Australian youth track and field athletes, and the notion that performance athletes in 'centimetres, grams, or seconds' sports increase their training practices during late adolescence $[13,19]$. However, no other significant differences were found between age-groups, in relation to the training practices of these athletes. This might be because a large proportion (58\%) of these adolescent distance runners had higher levels of specialisation and broadly similar training ages, regardless of sex.

The fact that both the volume and intensity of training practices do not differ much between age-groups is concerning, especially considering that distance running is a late-specialisation sport $[19,20]$. For example, the average number of training months per year (11.0) in this cohort exceeds current evidencebased recommendations, having been associated with injury history in youth athletes [22]. However, it is important to recognise that self-reported intensity was based on perceived exertion. Therefore, this does not mean that other physiological or biomechanical measures of intensity did not vary between participants and the different age-groups.

In further contradiction to the training recommendations [22], the exploratory analyses highlight that the more training sessions that an adolescent runner completes per week, were associated with a lower risk of injury. When only including time loss RRI, a higher level of specialisation was also associated with a lower risk of injury, compared to those with a lower level of specialisation. Whilst there are several confounding factors to consider when interpreting these results, they highlight that the current training 
recommendations may need revaluating for specific sports. However, analytic epidemiology is required to detail what this would involve within adolescent distance running, having quantified the association between training exposure and RRI.

\subsection{Running-Related Injuries:}

For "all RRI," the reported injury IP was high in this population (126/100 participants/year), when compared with other youth sport studies [33]. When considering exposure time, the reported injury IR (6.3/1000 participation hours), for "all RRI," was slightly higher than that found in a two different cohorts of elite Swedish adolescent distance runners (4.0 to 5.3/1000 participation hours) [8, 11]. Yet, these rates are lower than those found in recreational (7.7/1000 participation hours) and novice (17.8/1000 participation hours) adult runners [4]. Overall, these IP and IR results indicate that this cohort of competitive adolescent distance runners may have a greater training volume (exposure) than in other sports, whereby the higher IP may be a result of this greater training volume.

The injury IR for male participants (5.9/1000 participation hours), for "all RRI," is higher than that reported in several other youth sports, with a similar injury IR to that found in youth football studies [32]. A similar pattern was found in terms of the injury IR for female participants (6.6/1000 participation hours), indicating a lack of significant sex differences. This is further supported by a non-significant rate ratio between the male and female participants in this study (rate ratio $=0.89 ; 95 \% \mathrm{Cl}: 0.68-1.33$ ). Notably, the studies reporting injury IR in a population of youth cross-country runners calculated this outcome according to the number of injuries per 1000 athletic exposures, making comparison of results difficult [9, 10].

The injury IP for those RRI that required medical attention (52/100 participants/year) was higher than that reported in athletes aged 11-18 years (35/100 participants/year), across a range of sports [16]. The largest proportion of RRI incurring time loss were categorised as "serious" $(25 \%)$. Although this is an interesting finding, highlighting that a quarter of RRI resulted in more than 28-days of time loss (up to 6months), this may be due to recall bias. On the contrary, the large proportion of RRI incurring "no time loss" (22\%) was due to participants being able to register "any physical complaint" when self-reporting RRI, whereby $45 \%(n=30)$ of self-reported 'pains or discomforts' did not result in time loss.

RRI were most commonly reported in the lower extremity, with the knee, foot/toe and lower leg being the most frequently injured sites. These injury sites are comparable to those reported in elite adult and adolescent track and field athletes [17]. Likewise, the most common self-reported cause of injury was overuse, which supports previous findings [45]. These data indicate that injury prevention interventions for adolescent distance runners should predominantly focus on reducing the risk of lower extremity RRI caused by overuse.

\subsection{Methodological Limitations:}

The main limitation of this study is the use of a cross-sectional study design. As a result, it is not possible to determine temporal relationships between potential injury risk factors and RRI. Recruitment difficulties 
resulted in a limited sample size is, also considered a study limitation. While this is not a limitation for the descriptive data, the limited sample size meant that only exploratory univariate analyses were conducted when investigating potential correlates of RRI. The convenience sampling method may also have led to a non-representative sample.

Recall bias is a further study limitation, whereby the accuracy of data was dependent upon self-report. This type of bias often results in participants under-reporting minor injuries, leading to an artificially greater proportion of severe injuries. While there was a high proportion of serious time loss RRI, the proportion of RRI that incurred no time loss was also elevated. Nonetheless, research has shown that participants can accurately recall the total number of injuries and injury sites when providing a 12-month self-reported history [46]. However, as that research was based in a different sporting context, the effect of recall bias remains unclear within this study. Social desirability bias is also possible, whereby participants could have over-reported their training practices (frequency, volume, and intensity).

\subsection{Conclusions}

This study found that the injury IP for competitive adolescent distance runners is higher than that reported for other youth sports. The injury IR is slightly higher than studies that have included youth endurance athletes, but lower when compared to studies that only included adult runners. These results suggest that this higher IP could be due to a greater training volume (exposure) in competitive adolescent distance runners, when compared to other youth sports. The knee, foot/toe, and lower leg were the most common injury sites, with overuse being the most common cause of injury. There was a high level of specialisation within this population, and the total number of training sessions per week (frequency) increased with age. In relation to the risk of incurring RRI, a higher level of specialisation was associated with a lower risk of injury, compared to those with a lower level of specialisation. In relation to incurring a time loss injury, increased training frequency was associated with a lower risk of injury. However, due to the limited sample size, this exploratory univariate analysis needs to be further supported by analytical epidemiology. Therefore, a prospective cohort study should be conducted in order to establish temporal relationships between injury risk factors and RRI in this population.

\section{Abbreviations}

\%: Percentage

BMl: Body mass index

Cl: Confidence intervals

IOC: International Olympic Committee

IP: Incidence proportion 
IQR: Interquartile range

IR: Incidence rate

OR: Odds ratio

RPE: Rating of perceived exertion

RRI: Running-related injuries

SD: Standard deviations

SRPE: Session rating of perceived exertion

$\mathrm{X}^{2}$ : Chi-squared test

Declarations

ETHICS APPROVAL AND CONSENT TO PARTICIPATE

Written parental consent and participant assent were obtained before completion of the questionnaire. Ethics approval was granted by the Sport and Health Sciences ethics committee (171206/B/02), at the University of Exeter.

\section{CONSENT FOR PUBLICATION}

Not applicable.

\section{AVAILABILITY OF DATA AND MATERIALS}

Data are available from the corresponding author upon reasonable request.

\section{COMPETING INTERESTS}

No competing interests (financial or otherwise) declared.

\section{FUNDING}

This work was supported by the Economic and Social Research Council (ESRC) under the following grant: ES/J50015X/1. The ESRC was not involved with any element of conducting this research, from study design through to submitting this article for publication. 


\section{AUTHORS' CONTRIBUTIONS}

$R M, A B$ and $C W$ were involved in the design of the study. RM was responsible for study implementation. $R M$ and $A B$ did the statistical analyses. RM wrote the first draft of the manuscript, with $A B$ making initial revisions. All other revisions by RM were circulated and commented on by $A B, C W, B C$ and $C M$. All authors read and approved the final manuscript.

\section{ACKNOWLEDMENTS}

The authors appreciate and acknowledge the athletes, parents, coaches, and medical and healthcare practitioners involved in this research. Thanks to Morten W. Fagerland, for supporting data analysis. Robert H. Mann is supported by an Economic and Social Research Council PhD studentship (ES/J50015X/1), as part of the South West Doctoral Training Partnership. This PhD is in collaboration with England Athletics.

\section{References}

1. Hulteen RM, Smith JJ, Morgan PJ, Barnett LM, Hallal PC, Colyvas K, et al. Global participation in sport and leisure-time physical activities: A systematic review and meta-analysis. Preventive medicine. 2017;95:14-25.

2. Pedisic Z, Shrestha N, Kovalchik S, Stamatakis E, Liangruenrom N, Grgic J, et al. Is running associated with a lower risk of all-cause, cardiovascular and cancer mortality, and is the more the better? A systematic review and meta-analysis. Br J Sports Med. 2019:bjsports-2018-100493.

3. van Gent RN, Siem D, van Middelkoop M, van Os AG, Bierma-Zeinstra SMA, Koes BW. Incidence and determinants of lower extremity running injuries in long distance runners: a systematic review. $\mathrm{Br} J$ Sports Med. 2007;41(8):469-80.

4. Videbaek S, Bueno AM, Nielsen RO, Rasmussen S. Incidence of Running-Related Injuries Per $1000 \mathrm{~h}$ of running in Different Types of Runners: A Systematic Review and Meta-Analysis. Sports Med. 2015;45(7):1017-26.

5. Edouard P, Navarro L, Branco P, Gremeaux V, Timpka T, Junge A. Injury frequency and characteristics (location, type, cause and severity) differed significantly among athletics ('track and field') disciplines during 14 international championships (2007-2018): implications for medical service planning. $\mathrm{Br} \mathrm{J}$ Sports Med. 2019:bjsports-2019-100717.

6. Kluitenberg B, van Middelkoop M, Diercks R, van der Worp H. What are the Differences in Injury Proportions Between Different Populations of Runners? A Systematic Review and Meta-Analysis. Sports Med. 2015;45(8):1143-61.

7. Tabben M, Whiteley R, Wik E, Bahr R, Chamari K. Methods may matter in injury surveillance: "how" may be more important than "what, when or why". Biology of sport. 2019:3-5. 
8. von Rosen P, Floström F, Frohm A, Heijne A. Injury patterns in adolescent elite endurance athletes participating in running, orienteering, and cross-country skiing. Int J Sports Phys Ther. 2017;12(5):822-32.

9. Rauh MJ, Koepsell TD, Rivara FP, Margherita AJ, Rice SG. Epidemiology of musculoskeletal injuries among high school cross-country runners. Am J Epidemiol. 2006;163(2):151-9.

10. Rauh MJ, Margherita AJ, Rice SG, Koepsell TD, Rivara FP. High school cross country running injuries: a longitudinal study. Clinical journal of sport medicine: official journal of the Canadian Academy of Sport Medicine. 2000;10(2):110-6.

11. Rosen Pv, Heijne A, Frohm A, Fridén C, Kottorp A. High Injury Burden in Elite Adolescent Athletes: A 52-Week Prospective Study. J Athl Train. 2018;53(3):262-70.

12. Jacobsson J, Timpka T, Kowalski J, Nilsson S, Ekberg J, Renström P. Prevalence of Musculoskeletal Injuries in Swedish Elite Track and Field Athletes. The American Journal of Sports Medicine. 2012;40(1):163-9.

13. Huxley DJ, O'Connor D, Healey PA. An examination of the training profiles and injuries in elite youth track and field athletes. European journal of sport science. 2014;14(2):185-92.

14. Pierpoint LA, Williams CM, Fields SK, Comstock RD. Epidemiology of Injuries in United States High School Track and Field: 2008-2009 Through 2013-2014. Am J Sports Med. 2016;44(6):1463-8.

15. England S. Active Lives data tables 2019 [Available from: https://www.sportengland.org/know-youraudience/data/active-lives/active-lives-data-tables\#children_and_young_people_surveys.

16. Bergeron MF, Mountjoy M, Armstrong N, Chia M, Côté J, Emery CA, et al. International Olympic Committee consensus statement on youth athletic development. Br J Sports Med. 2015;49(13):84351.

17. Jacobsson J, Timpka T, Kowalski J, Nilsson S, Ekberg J, Renstrom P. Prevalence of musculoskeletal injuries in Swedish elite track and field athletes. Am J Sports Med. 2012;40(1):163-9.

18. Carragher P, Rankin A, Edouard PA, One-Season. Prospective Study of Illnesses, Acute, and Overuse Injuries in Elite Youth and Junior Track and Field Athletes. Frontiers in Sports and Active Living. 2019;1(13).

19. Moesch K, Elbe AM, Hauge ML, Wikman JM. Late specialization: the key to success in centimeters, grams, or seconds (cgs) sports. Scand J Med Sci Sport. 2011;21(6):e282-90.

20. Kearney PE, Hayes PR. Excelling at youth level in competitive track and field athletics is not a prerequisite for later success. Journal of sports sciences. 2018;36(21):2502-9.

21. Shibli S, Barrett D. Bridging the Gap... Research to Provide Insight into the Development and Retention of Young Athletes. Sheffield Sheffield Hallam University; 2011.

22. Post EG, Trigsted SM, Riekena JW, Hetzel S, McGuine TA, Brooks MA, et al. The Association of Sport Specialization and Training Volume With Injury History in Youth Athletes. Am J Sports Med. 2017;45(6):1405-12. 
23. Fabricant PD, Lakomkin N, Sugimoto D, Tepolt FA, Stracciolini A, Kocher MS. Youth sports specialization and musculoskeletal injury: a systematic review of the literature. Phys Sportsmed. 2016;44(3):257-62.

24. Myer GD, Jayanthi N, Difiori JP, Faigenbaum AD, Kiefer AW, Logerstedt D, et al. Sport Specialization, Part I: Does Early Sports Specialization Increase Negative Outcomes and Reduce the Opportunity for Success in Young Athletes? Sports Health. 2015;7(5):437-42.

25. Seiler $\mathrm{S}$. What is best practice for training intensity and duration distribution in endurance athletes? International Journal of Sports Physiology Performance. 2010;5(3):276-91.

26. Nielsen RO, Buist I, Sørensen H, Lind M, Rasmussen S. Training errors and running related injuries: a systematic review. Int J Sports Phys Ther. 2012;7(1):58-75.

27. Brewer BW, Van Raalte JL, Linder DE. Athletic identity: Hercules' muscles or Achilles heel? International Journal of Sport Psychology. 1993;24(2):237-54.

28. Woollings KY, McKay CD, Kang J, Meeuwisse WH, Emery CA. Incidence, mechanism and risk factors for injury in youth rock climbers. Br J Sports Med. 2015;49(1):44-50.

29. Soligard T, Schwellnus M, Alonso JM, Bahr R, Clarsen B, Dijkstra HP, et al. How much is too much? (Part 1) International Olympic Committee consensus statement on load in sport and risk of injury. $\mathrm{Br}$ J Sports Med. 2016;50(17):1030-41.

30. Timpka T, Alonso JM, Jacobsson J, Junge A, Branco P, Clarsen B, et al. Injury and illness definitions and data collection procedures for use in epidemiological studies in Athletics (track and field): consensus statement. Br J Sports Med. 2014;48(7):483-90.

31. Clarsen B, Bahr R. Matching the choice of injury/illness definition to study setting, purpose and design: one size does not fit all! Br J Sports Med. 2014;48(7):510-2.

32. Caine D, Maffulli N, Caine C. Epidemiology of injury in child and adolescent sports: injury rates, risk factors, and prevention. Clin Sports Med. 2008;27(1):19-50. vii.

33. Emery CA. Risk Factors for Injury in Child and Adolescent Sport: A Systematic Review of the Literature. Clinical journal of sport medicine: official journal of the Canadian Academy of Sport Medicine. 2003;13(4):256-68.

34. Cole TJ, Bellizzi MC, Flegal KM, Dietz WH. Establishing a standard definition for child overweight and obesity worldwide: international survey. Bmj. 2000;320(7244):1240-3.

35. Cole TJ, Flegal KM, Nicholls D, Jackson AA. Body mass index cut offs to define thinness in children and adolescents: international survey. Bmj. 2007;335(7612):194.

36. Moore SA, McKay HA, Macdonald H, Nettlefold L, Baxter-Jones AD, Cameron N, et al. Enhancing a Somatic Maturity Prediction Model. Med Sci Sports Exerc. 2015;47(8):1755-64.

37. Baxter-Jones ADG, Eisenmann JC, Sherar LB. Controlling for Maturation in Pediatric Exercise Science. 2005;17(1):18.

38. Jayanthi N, Pinkham C, Dugas L, Patrick B, Labella C. Sports specialization in young athletes: evidence-based recommendations. Sports Health. 2013;5(3):251-7. 
39. Jayanthi N, LaBella C, Fischer D, Pasulka J, Dugas L. Sports-specialized intensive training and the risk of injury in young athletes: a clinical case-control study. Am J Sports Med. 2015;43(4):794-801.

40. Mann RH, Williams CA, Clift BC, Barker AR. The Validation of Session Rating of Perceived Exertion for Quantifying Internal Training Load in Adolescent Distance Runners. International Journal of Sports Physiology Performance. 2019;14(3):354-9.

41. Knowles SB, Marshall SW, Guskiewicz KM. Issues in estimating risks and rates in sports injury research. J Athl Train. 2006;41(2):207.

42. Marshall S. Prevalence and Incidence. In: Kempf-Leonard K, editor. Encyclopedia of Social Medicine. 3. New York: Elsevier; 2005. pp. 141-7.

43. Greenland S, Rothman KJ. Introduction to Categorical Statistics. In: Rothman KJ, Greenland S, Lash TL, editors. Modern Epidemiology. 3rd ed. Philadelphia: Lippincott Williams \& Wilkins; 2008. pp. 238-57.

44. Cohen J. A power primer. Psychol Bull. 1992;112(1):155-9.

45. DiFiori JP, Benjamin HJ, Brenner JS, Gregory A, Jayanthi N, Landry GL, et al. Overuse injuries and burnout in youth sports: a position statement from the American Medical Society for Sports Medicine. Br J Sports Med. 2014;48(4):287-8.

46. Gabbe BJ, Finch CF, Bennell KL, Wajswelner $\mathrm{H}$. How valid is a self reported 12 month sports injury history? Br J Sports Med. 2003;37(6):545-7.

\section{Figures}

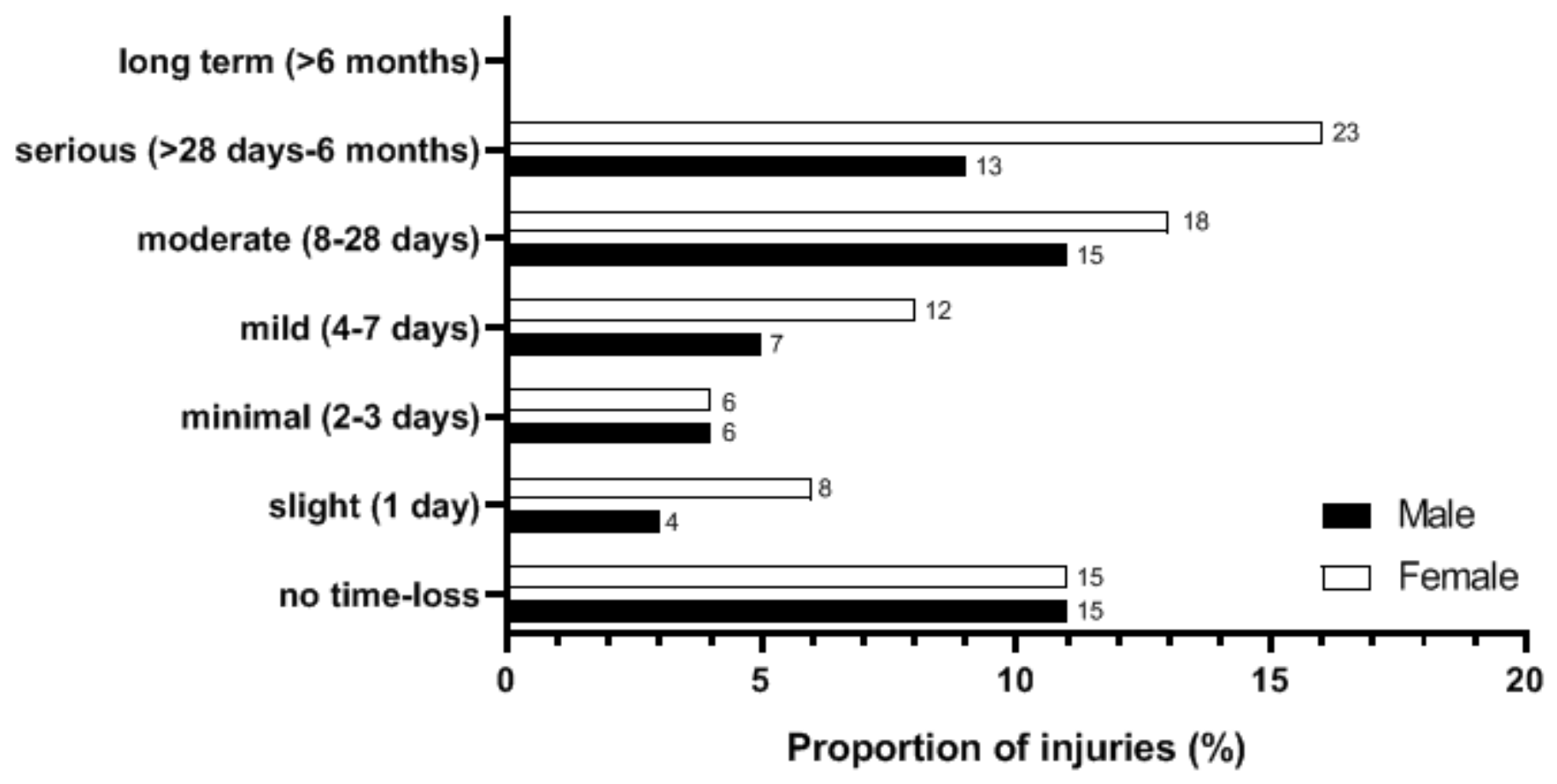

Figure 1 
Proportion of running-related injuries (RRI) incurring time loss from distance running in days. NB: Due to rounding, not all numbers add up correctly.

\section{Supplementary Files}

This is a list of supplementary files associated with this preprint. Click to download.

- StudyQuestionnaireSupplementaryFile.pdf 